\title{
Modified Location-Aided Routing Protocols for Control Overhead Reduction in Mobile Ad Hoc Networks
}

\author{
Sidi-Mohammed Senouci and Tinku Mohamed Rasheed \\ France Telecom R\&D \\ 2 Avenue Pierre Marzin, 22307 \\ Lannion, France \\ \{ sidimohammed.senouci, \\ tinku.mohamedrasheed\}@ francetelecom.com
}

\begin{abstract}
In geographical ad hoc routing, each node has to be equipped with Global Positioning System (GPS). This requirement is quite realistic today as such devices are inexpensive and can provide reasonable precision. In this work, we are interested in the optimization of the geographical routing protocol, LAR (Location-Aided Routing) [1]. LAR is an on-demand routing protocol using geographical location information to limit the area for discovering a new route to a smaller "request zone". Instead of flooding the route requests into the whole network, only nodes in the request zone will forward them. Thus, the routing overhead is widely reduced. In this paper, we compare some optimization of LAR. The proposed optimizations use alternative definitions of request zone by intermediate nodes. The simulation results show that these algorithms lead to an improvement in terms of routing overhead.
\end{abstract}

Keywords - ad hoc routing; location; GPS; LAR.

\section{Introduction}

A Mobile Ad-Hoc Network (MANET) is a collection of wireless mobile nodes forming a temporary network without using any centralized access point, infrastructure, or centralized administration. The wireless mobile hosts communicate in a multi-hop fashion. A set of ad hoc routing protocols have been proposed in the IETF's MANET [2]group to ensure the network connectivity. In

Please use the following format when citing this chapter:

Senouci, S.-M, and Rasheed, T.M., 2007, in IFIP International Federation for Information Processing, Volume 229, Network Control and Engineering for QoS, Security, and Mobility, IV, ed. Gaiti, D., (Boston: Springer), pp. $137-146$. 
[3], the author classifies these protocols into three categories: (i) flat routing schemes, which are further classified into two classes: proactive and reactive; (ii) hierarchical routing; and (iii) geographic position assisted routing. Flat routing approaches (like OLSR, TBRPF, AODV and DSR) adopt a flat addressing scheme. Each node participating in routing plays an equal role. In contrast, hierarchical routing (like CGSR, HSR and ZRP) usually assigns different roles to the network nodes. Some protocols require a hierarchical addressing system. Routing with the assistance from geographic location information (like LAR, GeoCast and GPSR) requires each node to be equipped with Global Positioning System (GPS). This requirement is quite realistic today as such devices are inexpensive and can provide reasonable precision. The author gives a summary of the scalable features of protocols in the three categories and with some future research direction.

In this work, we are interested in the optimization of the geographical routing protocol, LAR (Location-Aided Routing) [1][4]. The remainder of this paper is organized as follows. After a brief description of the basic LAR protocol in section 2, we review the related work in section 3 . In section 4 , an overview of the optimization schemes of LAR are given. The methodology used for the performance evaluations is exposed in section 5. Finally, section 6 summarizes the main contributions of this work.

\section{LAR Routing Protocol Basics}

LAR is an on-demand routing protocol whose operation is similar to DSR (Dynamic Source Routing) [5]. In contrast to DSR, LAR protocol uses geographical location information to limit the area for discovering a new route to a smaller "request zone". Instead of flooding the route requests into the entire network, only those nodes in the request zone will forward them.

To determine the request zone, there are two schemes. In the first one, the source estimates a circular area (expected zone) in which the destination is expected to be found at the current time. The position and the size of the circle are calculated based on the location knowledge of the previous destination, the time instant associated with the previous location record and the average speed of the destination (see Fig.1). The request zone is the smallest rectangular region that includes the expected zone and the source. The coordinates of the four corners are included in the route request packet when initiating the route discovery process. RREQ broadcast is limited to this request zone. Thus, when the node in the request zone receives $R R E Q$, it forwards the packet normally. However when a node which is not in the request zone receives an RREQ, it drops the packet. For example, in Figl, if node $I$ receives the route request from another node, node $I$ forwards the request to its neighbors, because $I$ determines that it is within the rectangular request zone. However, when node $J$ receives the route request, it discards the request, as node $J$ is not within the request zone. 


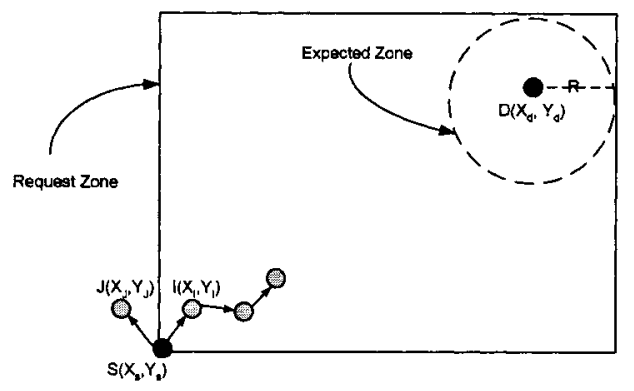

Fig. 1. Standard LAR scheme 1.

If source node $S$ knows a previous location of destination node $D$ at time $t_{0}$, if it also knows its average speed $v$ and the current time $t_{l}$, then the expected zone at time $t_{l}$ is a circle around $P$ with radius $R=v^{*}\left(t_{1}-t_{0}\right)$.

As soon as the destination $D$ receives the route request packet, it sends back a route reply packet as in the flooding algorithms. Its reply differs by containing its current position, the actual time, and its average speed. Source node $S$ is going to use this information for a route discovery in the future.

In the second scheme, the source calculates the distance to the destination based on the location of the destination. This distance, is included in the route request message and sent to neighbors. When an intermediate node receives the request, it calculates its distance to the destination. It will relay the request only if its distance to the destination is less than the distance included in the request message.

\section{Related Work}

Designing efficient routing protocols is an important research issue in mobile ad hoc networks. Many routing algorithms have been proposed to reduce route discovery overhead [6][7][8]. TORA (Temporally-Ordered Routing Algorithm) [6] is designed to reduce reaction to topological modification by localizing routing related messages to a smaller region near the change. On-Demand Multicast Routing Protocol (ODMRP) [7] is a multicast routing protocol based on a mesh topology and a forwarding group concept (i.e., only a subset of nodes forward the multicast packets). OLSR (Optimized Link State Routing) [8] reduces the control traffic overhead by using Multipoint Relays (MPR). An MPR is a node's one-hop neighbor which has been chosen to forward packets. Instead of pure flooding of the network, packets are just forwarded by a node's MPRs. This delimits the network overhead, thus being more efficient than pure link state routing protocols. Q-AOMDV (Q-routing for Ad hoc On-demand Multipath Distance Vector in ad hoc networks) [9] computes multiple paths in a single route discovery attempt. A 
new route discovery is required only when all paths to the destination break. Reviewing all these protocols is out of the scope in the present context.

Most of these MANET routing algorithms do not consider the physical location of a destination node. Recall that the principal goal of position-based routing protocols is to minimize the route discovery overhead by minimizing the number of forwarding nodes. For position-based routing protocols, if a source node wants to communicate with a destination node, it generally knows the position of its destination. Packets are forwarded to the next hop in the direction of the destination until they reach their destination. A set of position-based proposals, exploiting information about the geographic location of the mobile node, has emerged which improves the routing performances [10]. Among location-based routing protocols, we focus on the Location-Aided Routing (LAR) described in section 2. However, several optimizations are possible to achieve more efficient performance with the basic LAR protocols. In [1][4][11][12], some potential optimizations to the basic LAR algorithms have been suggested, for instance, alternative definitions of request zone or use of directional antenna, etc. In this section, we summarize them. Optimizations related to the definition of new request zones are detailed and evaluated in the following sections.

In [4], the authors propose an adapted request zone by intermediate nodes. Indeed, in standard LAR scheme 1, the requested zone is computed only by the source node. The adaptation of this zone by intermediate nodes, using more recent location information for destination host, can improve the probability of finding a route to the destination.

In [11] the authors suggest an approach where they suppose the existence of fixed hosts (or rarely moving hosts) in the network. If such fixed host, say node $P$ (post), exists in the request zone defined in LAR algorithm, then the route request is performed in two steps. At first, source node $S$ sends a route request to node $P$, and node $P$ forwards the request to the destination node $D$. Thus, the size of the request zone is reduced which results in reducing the route request overhead.

In [12] the authors propose a modified-LAR algorithm and examine three variants of it. All of them are based on the idea of enlarging the request zone, in case of failure of the route discovery phase, instead of resorting to flooding.

\section{Optimizations of LAR}

We have seen in section 3 , that several optimizations are possible to achieve more efficient performance of the basic LAR protocols. In this section, we deal with those related to alternative definitions of the request zone. These new schemes are evaluated in the next section.

As stated in section 2, an intermediate node will forward a route request packet, only if it belongs to the request zone. The request zone should contain the expected zone to reach the destination node $D$. In standard LAR scheme 1 , the sides of the rectangle are always parallel to the $X$ and $Y$ axes. 
Only this rectangular shaped request zone is implemented. However, other definitions may be used. For instance, it is possible to remove this restriction when defining the rectangular region: one side of the rectangle may be made parallel to the line connecting the location of source node $S$ to the previous location of $D$ (see Fig. 2).

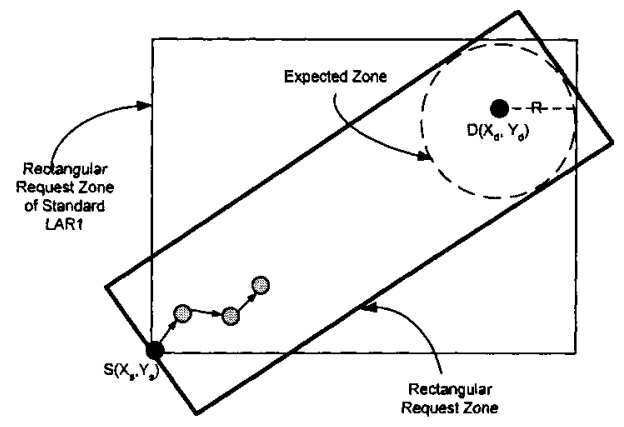

Fig. 2. Alternative definitions of request zone: tilted rectangular shaped.

In this scheme, the source node $S$ determines the coordinates of the four request zone vertices. These coordinates are relative to the plane where the node $S$ is the origin and the x-axis is parallel to the line connecting $S$ and $D$. Afterwards, the source translates these coordinates (for the four vertices) to the real coordinates using these formulae:

$$
\begin{aligned}
& x=x_{1} \times\left(y_{D}-y_{S}\right) / l+y_{1} \times\left(x_{D}-x_{S}\right) / l+x_{S} \\
& y=x_{1} \times\left(x_{s}-x_{d}\right) / l+y_{1} \times\left(y_{D}-y_{S}\right) / l+y_{S}
\end{aligned}
$$

Where $\left(x_{l}, y_{l}\right)$ are the coordinates of the vertex in the first plane, and $l$ is the distance between the source node $S$ and the destination node $D$. Hence, the coordinates of the four vertices area computed. These coordinates are included in the route request packet when initiating the route discovery process. RREQ broadcast is limited to this rectangular request zone. Thus, a node $I\left(x_{I}, y_{I}\right)$ forwards the RREQ packet only when it is in the request zone:

$$
\left\{\begin{array}{l}
x_{I} \geq \text { RequestZone.topLeft.x, and } \\
x_{I} \leq \text { RequestZone.bottomRight.x, and } \\
y_{I} \geq \text { RequestZone.bottomLeft.y, and } \\
y_{I} \leq \text { RequestZone.topRight.y }
\end{array}\right.
$$

As we can see in Fig. 3, the request zone can also be defined as a cone rooted at node $S$. When a node receives a route request, it discards the request if the node is not within the cone. A node $J$ is within the cone if it is either in the expected zone or in the triangle formed by the three lines $\left(\Delta_{l}, \Delta_{2}\right.$, and $\left.\Delta_{3}\right)$ : 


$$
\left\{\begin{array}{l}
J \in \text { Expected Zone }: \sqrt{\left(l-x_{J}\right)^{2}+y_{J}^{2}} \leq R \text {, or } \\
J \in \text { Triangle: } y_{J}-l-R \leq 0 \text { and } y_{J}-x_{J} \times l / R \geq 0 \text { and } y_{J}+x_{J} \times l / R \geq 0
\end{array}\right.
$$

Where $\left(x_{J}, y_{J}\right)$ are the coordinates of node $J$ in the first plane (plane where the node $S$ is the origin and the $x$-axis is parallel to the line connecting $S$ and $D$ ).

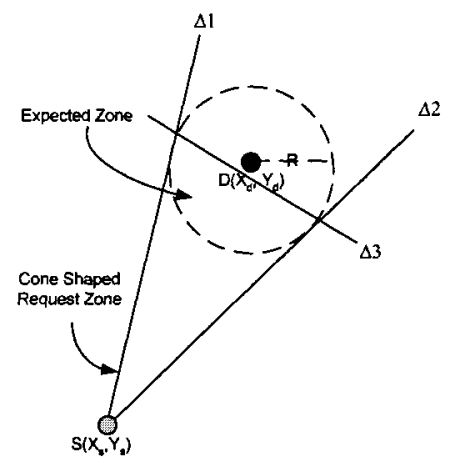

Fig. 3. Alternative definitions of request zone: cone shaped.

In contrast to the first scheme (titled rectangular shaped) where the source node $S$ includes the coordinates of the vertices of the request zone within the route request message, in the second scheme (cone shaped) only the radius information is transmitted within the RREQ messages when initiating a route discovery.

We can easily notice that these two approaches would often result in a smaller request zone than the standard LAR scheme 1 . Their performances are tested in section 5 .

\section{Experimental Results}

The performances of our algorithms are evaluated using QualNet simulator [13]. QualNet is the commercial version of the GloMoSim [14]. It is a discrete event, parallel simulation environment implemented in PARSEC [15]. Number of nodes in the network is chosen to be 20,30 and 50 for different simulation runs. The nodes are confined in a $1000 \times 1000 \mathrm{~m}^{2}$ area. Their initial locations are obtained using a uniform distribution. Individual nodes move following a random waypoint mobility model, and as in [1], each node moves continuously, without pausing at any location. We consider average speeds in the range of 1.5 to $22.5 \mathrm{~m} / \mathrm{s}$. A random connection is established using CBR traffic. As in [1], the source generates 10 data packets per second (on average), with a packet size of 64 bytes. In our simulations, standard IEEE 802.11 radios are adopted with channel rate as 2 
Mbps and transmission range as 300 meters. Simulation results are averaged over 10 runs, each with a different mobility pattern (different mobility patterns were obtained by choosing different seeds for a random number generator).

The performance metric is the fraction of routing packets per data connection, as a function of average speed. This is calculated as the ratio of the number of routing packets, and the number of data packets received by the destination.

Fig. 4, shows the performance comparisons by varying the average speed with 50,30 , and 20 nodes. As the number of nodes increases, the performance improvement of modified LAR1 becomes larger especially when the number of nodes is greater.

As the speed of mobile nodes increases, the routing overhead accumulates for all the routing protocols. With higher speed, the frequency of route breaking increases, thereby increasing the routing overhead to discover new routes. However, modified LAR1 schemes provide a lower routing overhead than standard LAR1 especially for higher speed. This is due to reduction of the number of route requests by limiting route discovery to a smaller request zone. As can be seen from the graph, cone shaped optimization has the smallest number of routing packets per route discovery since it has the smallest request zone.

Besides, with lower speed, the new schemes do not perform much better than standard LAR1 especially for a low network density (20 nodes). In order to explain this, recall that the radius of the expected zone for these simulations is obtained using the average speed of the destination node. The size of this zone is very significant for LAR and especially for its two variants. Indeed, with lower node velocities, the request zones become smaller and the probability of finding a route becomes increasingly difficult. The case is more complicated with a sparsely dense ad hoc network where there are not enough nodes in the request zone. Here, the number of neighbors for each node decreases especially for the cone shaped scheme. This factor affects the probability of a route discovery within the timeout interval, using the initial request zone. Recall that, in this case, LAR schemes allow the sender to initiate a new route discovery using the flooding algorithm. We believe that this is the reason why modified LAR schemes do not perform too well when network density is small.

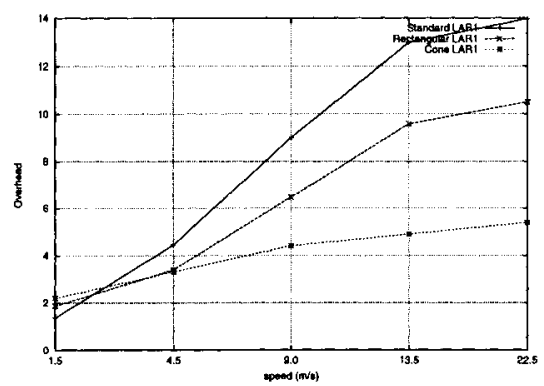

(a) 


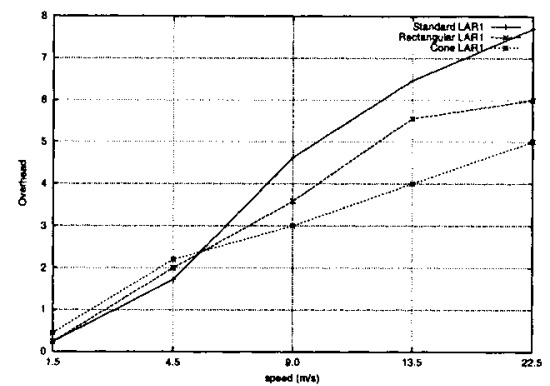

(b))

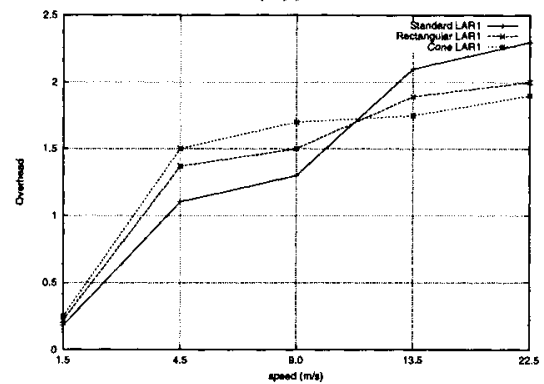

(c)

Fig. 4. Routing overhead versus average speed for: (a) 50 nodes, (b) 30 nodes, and (c) 20 nodes.

\section{Conclusion}

This paper describes how the basic LAR may be optimized to improve its performance. The proposed algorithms limit the request zone which results in reducing the route request overhead. Simulation results indicate that we can reduce the routing overhead using alternative definition of request zone as compared to LAR algorithm especially for dense and highly dynamic ad hoc networks. Several other optimizations are possible to achieve more efficient performance of the basic LAR (enlarging the request-zone area after the failure of a route-request rather than using the flooding algorithm, adaptation of the requestzone area according to node density, using directional antenna, etc.) and are intended to be dealt with and evaluated in the future works. 


\section{References}

1 Y.-B. Ko and N. H. Vaidya, "Location-aided routing (LAR) in mobile ad hoc networks," in ACM/IEEE International Conference on Mobile Computing and Networking (MobiCom'98), pp. 66-75, October 1998.

2 IETF MANET WG (Mobile Ad hoc NETwork), www.ietf.ora/html.charters/manet-charter.html.

3 X. Hong, K. Xu, and M. Gerla, "Scalable Routing Protocols for Mobile Ad Hoc Networks," IEEE Network, pp. 11-21, July/August 2002.

4 Y.-B. Ko and N. H. Vaidya, "Location-aided routing in mobile ad hoc networks," Tech. Rep. 98-012, CS Dept., Texas A\&M University, June 1998.

5 D. B. Johnson, D. A. Maltz, Y.-C. Hu, "The Dynamic Source Routing Protocol for Mobile Ad Hoc Networks (DSR)", IETF Internet Draft, draft-ietf-manet-dsr-09.txt.

6 V. D. Park and M. S. Corson, "Temporally Ordered routing algorithm (TORA) version1: functional specification", IETF Internet-Draft, August 1998.

7 M. Gerla, G. Pei, S. J, Lee, and C-C.Chiang, "On-Demand Multicast Routing protocol (ODMPR) for Ad hoc networks", IETF InternetDraft, November 1998.

8 T. Clausen and P. Jacquet, "Optimized Link State Routing Protocol (OLSR), IETF, RFC 3626, October 2003.

9 S. Senouci, "Minimization of Energy Consumption in Ad Hoc Networks", Annals of telecommunications, vol. 60, no 3-4, pp. 505$523,2005$.

10 M. Mauve, I. Widmer, H. Hartestein, "A Survey on Position-Based Rouitng in Mobile Ad Hoc Nehvorks", IEEE Network, November 2001.

$11 \mathrm{~J} .-\mathrm{K}$. Jeong and J.-H. Kim, "Performance Improvement of LocationAided Routing Using Fixed Post in Mobile Ad Hoc Networks", PDPTA 2000, Las Vegas, Nevada, USA, June 24-29, 2000.

12 F. De Rango, A. Iera, A. Molinaro, S. Marano, "A Modified LocationAided Routing Protocol for the Reduction of Control Overhead in Adhoc Wireless Networks", ICT'2003, vol.2, pp.1033-1037, February 23 - March 1, 2003

13 QualNet by Scalable Networks Technologies, http://www.qualnet.com.

14 X. Zeng, R. Bagrodia, and M. Gerla, "GloMoSim: a Library for Parallel Simulation of Large-scale Wireless Networks", In Proceedings of the 12th Workshop on Parallel and Distributed 
146 Modified Location-Aided Routing Protocols for Control Overhead Reduction in Mobile Ad Hoc Networks

Simulations (PADS'98), Banff, Alberta, Canada, pp. 154-161, May 26-29, 1998.

15 R. Bagrodia, R. Meyer, M. Takai, Y. Chen, X. Zeng, J. Martin, and H.Y. Song, "PARSEC: A parallel simulation environment for complex system," IEEE Computer Magazine, vol. 31, no. 10, pp. 7785 , October 1998. 